\title{
Interferon- $\beta$ sensitizes human glioblastoma cells to the cyclin-dependent kinase inhibitor, TG02
}

\author{
BIRTHE LOHMANN ${ }^{*}$, EMILIE LE RHUN* , MANUELA SILGINER, MIRKA EPSKAMP and MICHAEL WELLER \\ Laboratory of Molecular Neuro-Oncology, Department of Neurology, University Hospital and \\ University of Zurich, CH-8091 Zurich, Switzerland
}

Received June 25, 2019; Accepted September 30, 2019

DOI: $10.3892 / \mathrm{ol} .2020 .11362$

\begin{abstract}
Novel treatments for glioblastoma, the most common malignant primary brain tumor, are urgently required. Type I interferons (IFN) are natural cytokines primarily involved in the defense against viral infections, which may also serve a role in the control of cancer, notably in the suppression of the cancer stem cell phenotype. TG02 is a novel orally available cyclin-dependent kinase 9 inhibitor which induces glioma cell apoptosis without profound caspase activation, which is currently explored in early clinical trials in newly diagnosed and recurrent glioblastoma. In the present study, human glioma-initiating cell line models were used to explore whether IFN- $\beta$ modulates the anti-glioma activity of TG02. The present study employed immunoblotting to assess protein levels, several viability assays and gene silencing strategies to assess gene function. Pre-exposure to IFN- $\beta$ sensitized human glioma models to a subsequent exposure to TG02. Combination treatment was associated with increased DEVD-amc cleaving caspase activity that was blocked by the anti-apoptotic protein, BCL2. However, BCL2 did not protect from the synergistic effects of IFN and TG02 on glioma cell growth. Furthermore, although IFN strongly induced pro-apoptotic XIAP-associated factor (XAF) expression, disrupting XAF expression did not
\end{abstract}

Correspondence to: Professor Michael Weller, Laboratory of Molecular Neuro-Oncology, Department of Neurology, University Hospital and University of Zurich, Frauenklinikstrasse 26, CH-8091 Zurich, Switzerland

E-mail: michael.weller@usz.ch

${ }^{*}$ Contributed equally

Abbreviations: AnxV, AnnexinV; ARF1, ADP-ribosylation factor 1; CDK, cyclin-dependent kinase; GIC, glioma-initiating cell; HPRT1, hypoxanthine phosphoribosyltransferase 1; IFN, interferon; IFNAR, IFN- $\alpha / \beta$ receptors; MxA, myxovirus resistance protein $A ; P E$, phycoerythrin; pRNAPII, phosphorylated RNA polymerase II; PI, propidium iodide; RT-PCR, reverse transcriptase polymerase chain reaction; si, small interfering; XAF, XIAP-associated factor

Key words: cyclin-dependent kinase, glioma, synergy, targeted, therapy abrogate the synergy with TG02. Consistent with that, caspase 3 gene silencing did not abrogate the effects of TG02 or IFN- $\beta$ alone or in combination. Finally, it was observed that IFN- $\beta$ may indeed modulate the effects of TG02 upstream in the signaling cascade since inhibition of RNA polymerase II phosphorylation, a direct readout of the pharmacodynamic activity of TG02, was facilitated when glioma cells were pre-exposed to IFN- $\beta$. In summary, these data suggest that type I IFN may be combined with TG02 to limit glioblastoma growth, but that the well characterized effects of IFN and TG02 on apoptotic signaling are dispensable for synergistic tumor growth inhibition. Instead, exploring how IFN signaling primes glioma cells for TG02-mediated direct target inhibition may help to design novel and effective pharmacological approaches to glioblastoma.

\section{Introduction}

Glioblastoma is the most common malignant intrinsic brain tumor in adults (1). These tumors are believed to originate from molecular genetic lesions in neuroglial stem or progenitor cells and can arise at any age, but the risk of developing glioblastoma increases with age (2). The WHO classification characterizes glioblastomas as glial tumors exhibiting the histopathological features of microvascular proliferation and necrosis which are characteristics of WHO grade IV tumors (3). Typical glioblastomas do not show mutations in the isocitrate dehydrogenase genes, but genetic alterations in three major pathways: The phosphoinositide 3-kinase (PI3K)/protein kinase B (AKT)/mammalian target of rapamycin (mTOR), the p53 and the retinoblastoma (RB) pathways; furthermore, tyrosine kinases such as epidermal growth factor receptor and c-MET mediate and maintain the malignant phenotype of glioblastoma (4).

There has been essentially no progress in the pharmacological therapy of this tumor since the introduction of temozolomide in $2005(5,6)$. Notably targeted therapies have failed at least in unselected patient populations that were not pre-enriched for certain molecular markers (7). Type I interferons (IFN) have already been tested for potential anti-glioma activity in the early periods of cancer immunotherapy. IFN are endogenous cytokines that have a major role in combatting viral infections. Moreover, there is increasing interest in a role of type I IFN in suppressing tumor growth, 
notably a role in negatively regulating the cancer stem cell phenotype (8). We and others have previously characterized profound anti-glioblastoma stem cell properties of type I IFN in vitro that involved suppression of sphere formation but relatively little induction of cell death. The failure of IFN to induced cell death was surprising since the IFN-induced transcriptional changes in glioma cells were predicted to favour activation of the intrinsic caspase-dependent cell death pathway $(9,10)$. These promising cell culture data indicating efficacy of IFN against glioblastoma appear to be in conflict with the failure to confirm activity of type I IFN in clinical trials with human glioblastoma patients (11). Yet, interest in IFN signaling in glioblastoma persists and current efforts focus on integrating IFN into combined modality treatments.

TG02 is a novel orally available inhibitor of multiple cyclin-dependent kinases (CDK) that exhibits strong in vitro activity in glioma models while single agent activity in rodent glioma models remains moderate $(12,13)$. TG02 is currently explored in clinical trials in recurrent and in newly diagnosed glioblastoma (NCT02942264, NCT03224104) (14). The present study sought to explore whether the combination of type I IFN with TG02 might exhibit synergistic activity and might be suitable to activate caspase-dependent cell death pathways in human glioma models in vitro.

\section{Materials and methods}

Reagents. Human IFN- $\beta 1$ a was provided by Biogen Inc. Stock solutions for in vitro experiments were prepared in $20 \mathrm{mM}$ sodium acetate, $\mathrm{pH} 8.4$, containing $150 \mathrm{mM}$ arginine hydrochloride. TG02 was provided by Adastra. Staurosporine was purchased from AppliChem, acetyl-Asp-Glu-Val-Asp-7-ami no-4-methyl coumarin (ac-DEVD-amc) and benzyloxycarbonyl-Val-Ala-Asp (OMe)-fluoromethylketone (zVAD-fmk) were obtained from Bachem. ON-TARGET plus human siRNA SMART pool targeting human X-linked inhibitor of apoptosis protein (XIAP)-associated factor (XAF)-1 or caspase 3 was purchased from Dharmacon.

Cell culture. The human long-term cell line, LN-229, and the human glioma-initiating cell lines (GIC), T-325, ZH-161, $\mathrm{S}-24$, and $\mathrm{ZH}-305$, and their sensitivity to TG02 have been described in detail (12). LN-229 cells were cultured in Dulbecco's modified Eagle's medium supplemented with $10 \%$ fetal calf serum (Invitrogen) and $1 \%$ glutamine (Invitrogen). After approval of the local ethics committees of the procedure and after obtaining informed consent from patients, GIC were isolated from freshly resected tumors using the Papain Dissociation System (Worthington Biochemical Corporation). GIC were maintained in Neurobasal Medium (NB) with B-27 supplement $(20 \mu \mathrm{l} / \mathrm{ml})$ (Thermo Fisher Scientific, Inc.), L-glutamine $(10 \mu \mathrm{l} / \mathrm{ml})$, fibroblast growth factor-2 and epidermal growth factor (20 ng/ml each; Peprotech) and penicillin/streptomycin (pen-strep, Sigma-Aldrich/Merck). The GIC were studied within a range of maximum passages of 40-50. All cells were sent for short tandem repeat analysis (DSMZ) and are regularly tested for mycoplasma contamination, last in November 2018.
Acute growth inhibition assay. After seeding cells and after a recovery time of $24 \mathrm{~h}$ in complete medium, the cells were exposed to TG02 for acute growth inhibition assays for $72 \mathrm{~h}$ (high seeding density, $1.5 \times 10^{4}$ cells for LN-229 or $10^{4}$ cells for GIC per well) or for the determination of clonogenic survival (low seeding density, 50 cells for LN-229 or 150 cells per well for GIC) for more than ten days in 96 well plates. Human IFN- $\beta 1$ a was added $24 \mathrm{~h}$ prior to TG02 exposure and maintained throughout the experiment as indicated. Metabolic activity was assessed by MTT assay (Sigma Aldrich).

$R T$ - $q P C R$. For gene expression analyses, total mRNA was extracted after $24 \mathrm{~h}$ incubation in serum-free medium. Gene expression was determined by the Real-Time PCR System QuantStudio 6 using PowerUp SYBR ${ }^{\text {TM }}$ Green Master Mix (Thermo Fisher Scientific, Inc.) using primers at optimized concentrations. Relative quantification was calculated using the $\Delta$ CT-method (15) and specific target gene expression was normalized to the housekeeping genes hypoxanthine phosphoribosyltransferase 1 (HPRT1) or ADP-ribosylation factor 1 (ARF1). The following human-specific primers were used: IFNAR2 (Bio-Rad; qHsaCED0045841), XAF-1 (Bio-Rad; qHsaCED0045841), ARF1 (forward 5'-GACCAC GATCCTCTACAAGC-3', reverse 5'-TCCCACACAGTGAAG CTG-3'), caspase 3 (forward 5'-TGGAGATCAGCTCCCGAG ATG-3', reverse 5'-ATTGCCCACAGCCACTCTG-3'), HPRT1 (forward 5'-TGAGGATTTGGAAAGGGTGT-3', reverse 5'-AGCACACAGAGGGCTACAA-3'), IFNAR1 (forward 5'-TATGCTGCGAAAGTCTTCTTGAG-3', reverse 5'-TCT TGGCTAGTTTGGGAACTGTA-3') and myxovirus resistance protein A (MxA) (forward 5'-TGGAGATCAGCTCCC GAGATG-3', reverse 5'-ATTGCCCACAGCCACTCTG-3') (all Microsynth).

Immunoblotting. Immunoblot analysis was performed as described (12). Whole cell lysates were prepared using radioimmunoprecipitation assay (RIPA) buffer $[10 \mathrm{mM}$ Tris $\mathrm{pH} 8.0,150 \mathrm{mM} \mathrm{NaCl}, 1 \% \mathrm{NP}-40,0.5 \%$ deoxycholate, $0.1 \%$ SDS] supplemented with $1 \%$ complete protease inhibitor mix (Roche Diagnostics) and phosphatase inhibitor cocktails 1 and 2 (Sigma-Aldrich), and protein levels were determined by Bradford assay. After loading equal protein amounts, SDS-PAGE (10\% acrylamide gels) was performed under reducing conditions followed by protein transfer to nitrocellulose membranes. To avoid unspecific antibody binding, membranes were blocked in Tris-buffered saline containing $0.1 \%$ Tween 20 and $5 \%$ skim milk or $5 \%$ bovine serum albumin. For primary antibody incubation the following antibodies were used: Rabbit anti-IFNAR1 (LifeSpan BioSciences Inc., Seattle, WI, LS-C185508), mouse anti-MxA hybridoma supernatant (provided by J. Pavlovic, Zurich, Switzerland, clone 143), rabbit anti-XAF-1 (Santa Cruz Biotechnology; sc-19194), rabbit anti-caspase 3 (Santa Cruz Biotechnology; sc-9662), rabbit anti-phosphorylated-Rpb1 (RNAPII) (Cell Signaling Technology, 13499), rabbit anti-MCL-1 Cell Signaling Technology; 5453), rabbit anti-c-MYC (Cell Signaling Technology; 5605), rabbit anti-CDK9 (Santa Cruz Biotechnology; sc-13130) or rabbit anti- $\beta$-actin (Santa Cruz Biotechnology; sc1616). As secondary antibodies Amersham ECL sheep anti-mouse IgG-HRP (GE Healthcare 

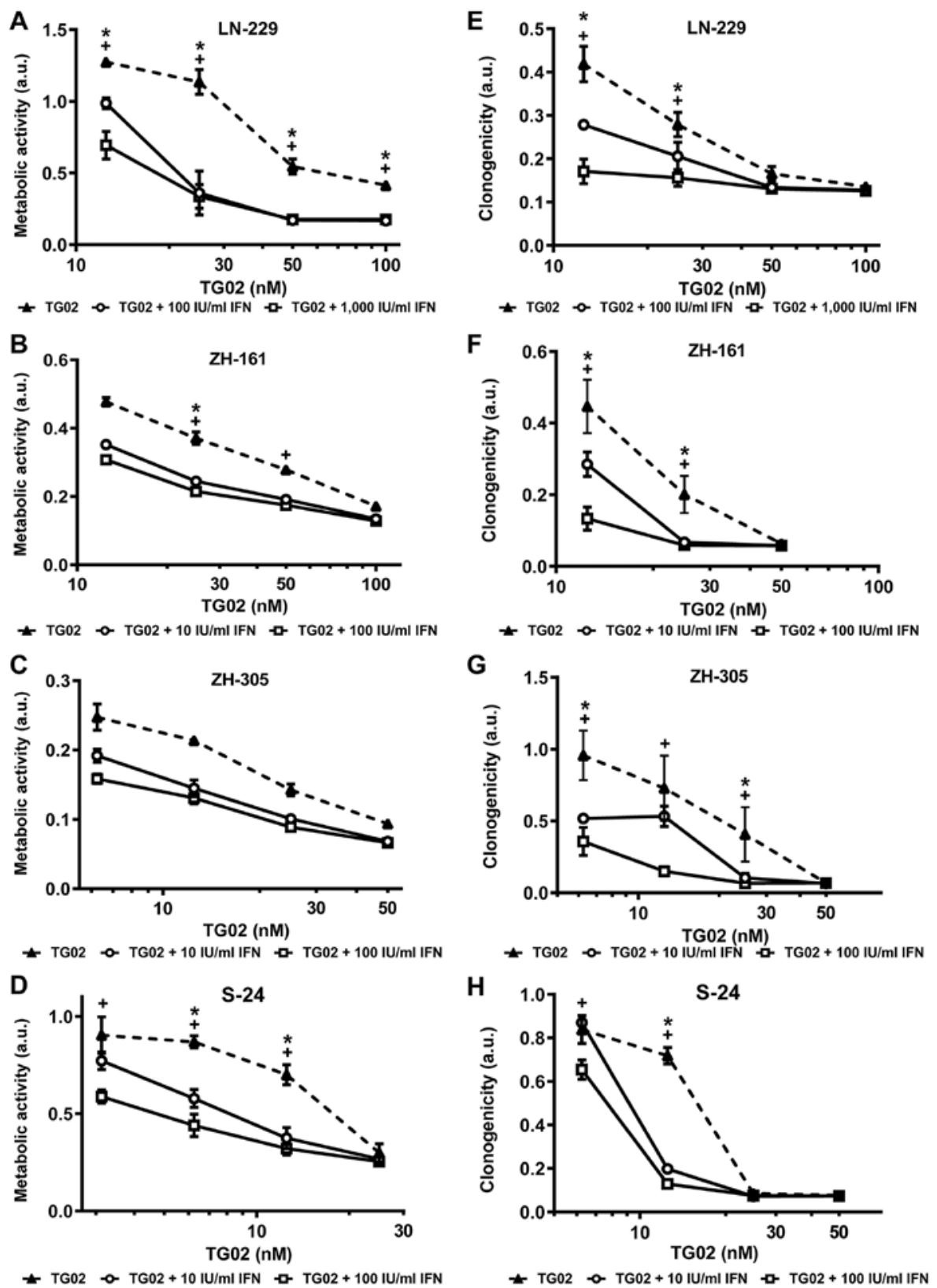

Figure 1. IFN- $\beta$ sensitizes human glioma cells to TG02. (A and E) LN-229, (B and F) ZH-161, (C and G) ZH-305 or (D and H) S-24 cells were pre-exposed or not to IFN- $\beta$ at 100 or $1,000 \mathrm{IU} / \mathrm{ml}$ for $\mathrm{LN}-229$ or 10 or $100 \mathrm{IU} / \mathrm{ml}$ for the three GIC models for $24 \mathrm{~h}$ and then exposed to increasing concentrations of TG02 in $72 \mathrm{~h}$ (A-D) acute growth inhibition or (E-H) clonogenic survival assays. Data are presented as mean percentages and standard error of the mean (n=3) of metabolic activity in a.u. relative to vehicle-treated control. "Synergy between TG02 and $100 \mathrm{IU} / \mathrm{ml}$ IFN- $\beta$ in LN-229 or 10 IU/ml IFN- $\beta$ in GIC. ${ }^{+}$Synergy between TG02 and 1,000 IU/ml IFN- $\beta$ in LN-229 or 100 IU/ml IFN- $\beta$ in GIC. a.u., arbitrary units; GIC, glioma-initiating cell; IFN, interferon.

Lifesciences; NA931V) or goat anti-rabbit IgG-HRP (Santa Cruz Biotechnology; sc-2004) were used.

Flow cytometry. Flow cytometry was performed according to standard protocols using phycoerythrin (PE)-conjugated monoclonal mouse anti-human IFNAR2 (PBL Assay Science; clone

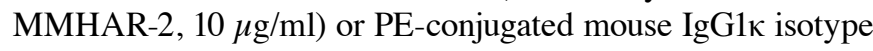
control (BD Biosciences; clone MOPC-21, $10 \mu \mathrm{g} / \mathrm{ml}$ ). Induction of cell death was analyzed using annexin $\mathrm{V}(\mathrm{AnxV})$ (BioLegend) and propidium iodide (PI) (Sigma-Aldrich) staining. Cells were washed with annexin buffer (10 mM HEPES, $140 \mathrm{mM}$ $\mathrm{NaCl}, 2.5 \mathrm{mM} \mathrm{CaCl}_{2}, \mathrm{pH}$ 7.4) and stained with $5 \mu \mathrm{l} \mathrm{AnxV}$ and $5 \mu \mathrm{l}$ PI [50 $\mu \mathrm{g} / \mathrm{ml}]$ for $15 \mathrm{~min}$ at room temperature in the dark. Staurosporine was used as positive control for apoptosis. Fluorescence intensity was recorded using a BD FACS Verse flow cytometer (BD Biosciences). Data (10,000 events per condition) were analyzed and median fluorescent intensities were calculated using FlowJo Version 10.0.7 (Tree Star).

Caspase activity assay. After exposure to TG02 or IFN-31a alone or in combination in the absence or presence of zVAD-fmk, the cells were lysed with lysis buffer followed by incubation with the fluorescent substrate Ac-DEVD-amc [6.25 $\mu \mathrm{M}]$. Measurement of DEVD-amc cleaving activity was performed using a Tecan Infinite M200 Pro plate reader (Tecan Trading AG) (480 nm emission; $360 \mathrm{~nm}$ excitation (12). 

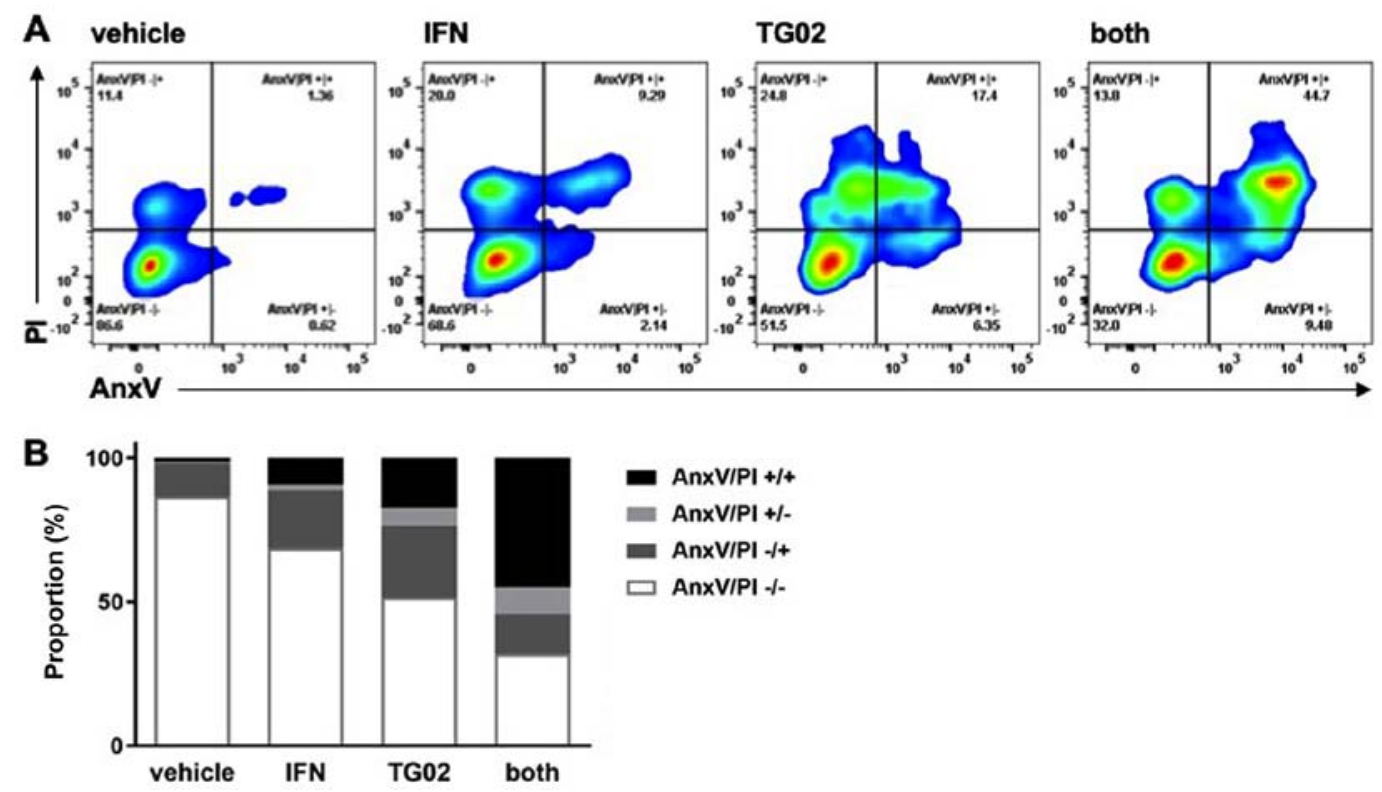

Figure 2. IFN- $\beta$ sensitizes human glioma cells to TG02. LN-229 cells were stimulated for $72 \mathrm{~h}$ with TG02 (100 nM) after being pre-exposed to IFN- $\beta$ $(1,000 \mathrm{IU} / \mathrm{ml})$ or not. (A) Cells were then subjected to AnxV/PI flow cytometry. (B) Proportional distribution of AnxV/PI fractions (AnxV/PI +/+ in black, AnxV/PI +/-in light grey, AnxV/PI-/+ in dark grey, AnxV/PI -/-in white) are indicated as bar graphs. AnxV, annexin V; PI, propidium iodide; IFN, interferon.

Transfection. For transient gene silencing, cells were transfected at $20 \mu \mathrm{M}$ of specific or scramble control small interfering (si) RNA by electroporation. Control or ON-TARGET plus siRNA SMARTpool were purchased from Dharmacon (caspase 3, L-004307-00-0005; xaf, L-004357-00-0005). At $72 \mathrm{~h}$ post transfection, samples were treated with IFN- $\beta 1 \mathrm{a}$ or TG02 or both as indicated and processed for further treatment. For stable gene overexpression, cells were transfected with the sequence-verified human bcl-2/pcDNA3 plasmid (Addgene no. 19279) using the X-tremeGENE ${ }^{\mathrm{TM}}$ HP DNA Transfection Reagent. G418 sulfate was used for selection of clones with transgene expression (12).

Statistical analysis. Data are representative of experiments performed two to three times with similar results. Statistical analysis was performed using GraphPad Prism 5 or 7 software. Statistical significance was assessed using either two-sided unpaired and paired Student's t-test or one-way ANOVA with Tukey's post hoc test for multiple comparison analysis. Quantitative data are represented as mean \pm standard deviation or standard error of the mean. $\mathrm{P}<0.05$ was considered to indicate a statistically significant difference. Synergy was assessed using the fractional product method (16) and differences exceeding 10\% of observed versus predicted (additive) effect were considered to indicate synergy.

\section{Results}

IFN- $\beta$ promotes TG02-induced cell death. The expression of IFN- $\alpha / \beta$ receptors 1 and 2 in the human glioma cell line models used here and their uniform responsiveness to IFN- $\beta$ in terms of MxA gene induction have been partially published $(10,17)$ and are summarized in Fig. S1. Growth inhibitory effects of TG02 alone in the cell line models studied here have also been characterized (12). We first explored whether pre-exposure to IFN- $\beta$ sensitized glioma cells to TG02 in short-term growth inhibition or clonogenicity respectively spherogenicity assays. GIC were exposed to lower concentrations of IFN- $\beta$ than the LN-229 long-term cell line because of the higher intrinisic sensitivity to IFN- $\beta$ of GIC (10). We noted strong sensitization by IFN- $\beta$ to TG02 in LN-229 and S-24 cells in acute growth inhibition assays and in S-24 cells in spherogenicity assays and largely additive effects in both assays in the other model systems (Fig. 1A-H). Synergistic induction of cell death by IFN- $\beta$ and TG02 in LN-229 cells was confirmed by AnxV/PI flow cytometry (Fig. 2).

Synergistic induction of caspase activation does not mediate synergistic growth inhibition by IFN- $\beta$ and TG02. We had previously observed that exposure to IFN- $\beta$ induced changes in gene expression, notably induction of xaf expression, predicting enhanced vulnerability to caspase-dependent apoptosis (10) and that TG02 alone induces largely caspase-independent apoptosis (12). Indeed, pre-exposure of LN-229 cells to IFN- $\beta$ facilitated processing of caspase 3 in response to TG02 as confirmed by immunoblot, albeit only to a minor degree compared with exposure to staurosporine which was used as a positive control (18) (Fig. 3A). Facilitation of induction of DEVD-amc-cleaving caspase activity by pre-exposure to IFN- $\beta$ was confirmed by a fluorimetric assay. Specificity was confirmed by demonstrating the abrogation of this caspase activity by the broad spectrum caspase inhibitor, zVAD-fmk, under all conditions (Fig. 3B). Similarly, BCL-2 gene transfer into LN-229 cells abrogated the promotion of caspase activation induced by the combination of IFN- $\beta$ and TG02, again using staurosporine as a positive control (Fig. 3C). We have previously reported that BCL-2 moderately attenuates TG02-induced cell death, likely in a caspase-independent manner since zVAD-fmk had no such effect except for high TG02 concentrations in selected models (12). Here we report that neither zVAD-fmk 
A

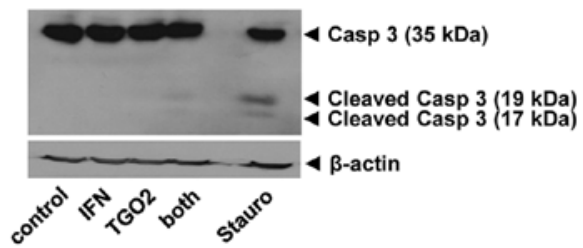

B
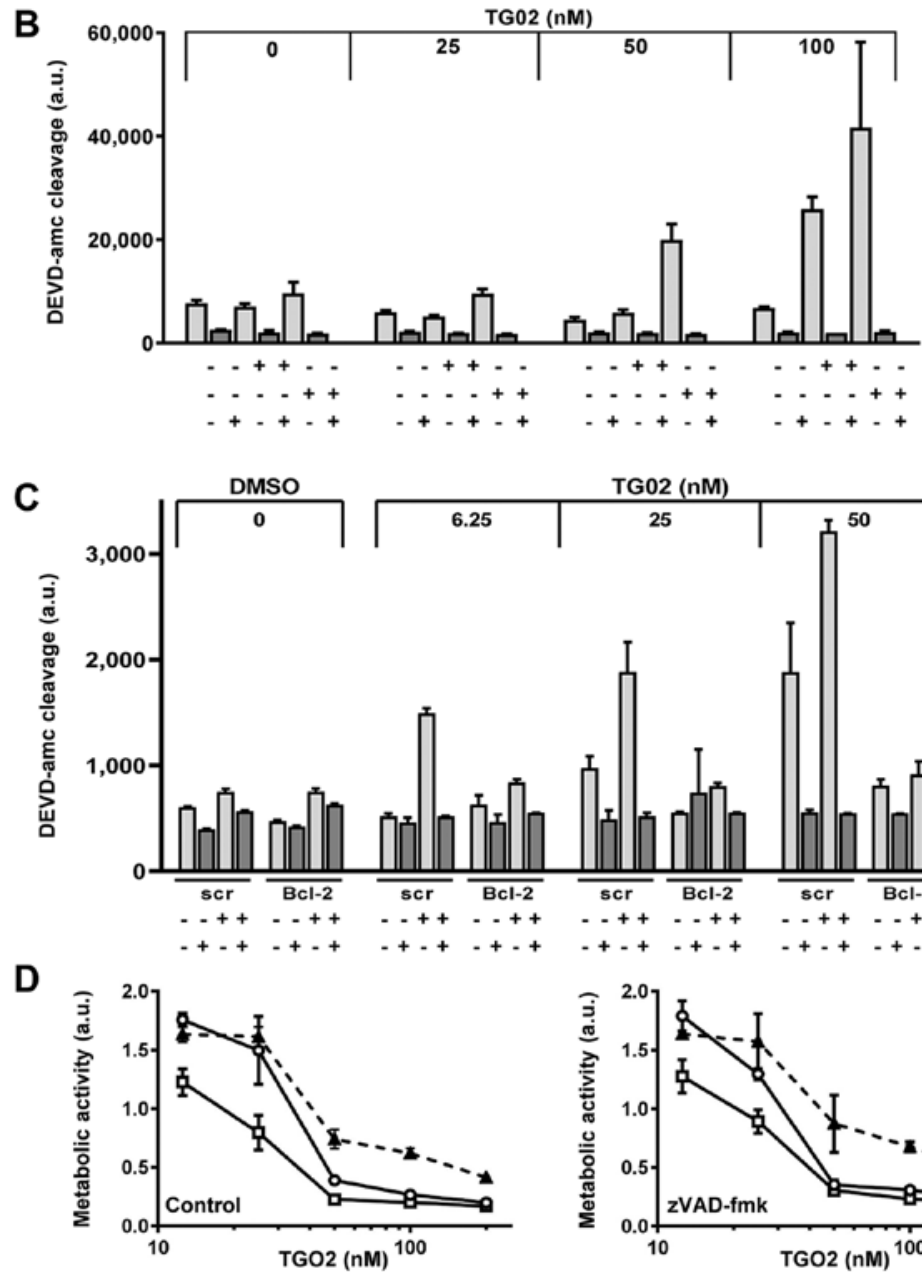

E

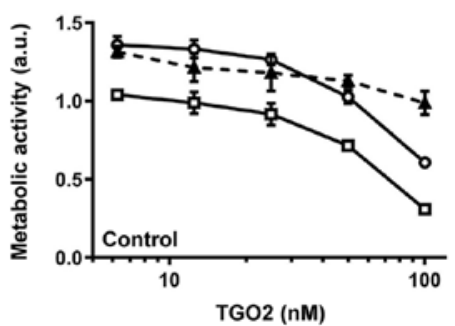

TG02 (nM)
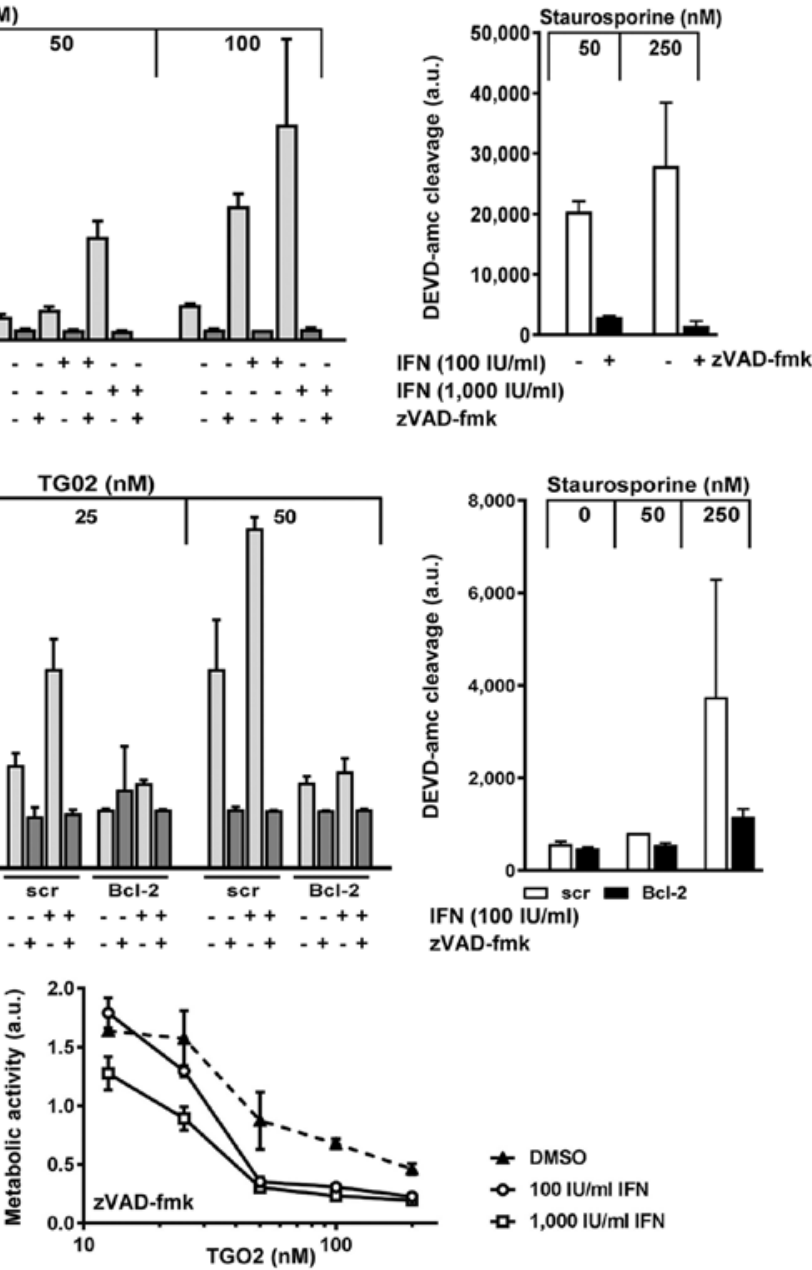

\pm DMSO

- $100 \mathrm{IU} / \mathrm{ml} \mathrm{IFN}$

- ㅁ. 1,000 IU/ml IFN

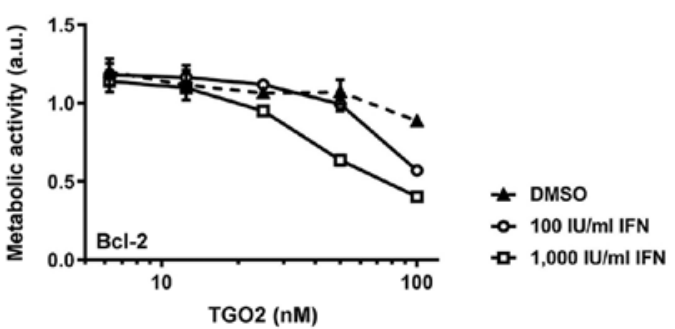

Figure 3. IFN- $\beta$ promotes DEVD-amc-cleavage activity induced by TG02. (A) Caspase 3 processing was monitored in LN-229 cells pre-exposed or not to IFN- $\beta$ $(1,000 \mathrm{IU} / \mathrm{ml})$ for $24 \mathrm{~h}$ and then treated with TG02 $(50 \mathrm{nM})$ in the absence or presence of IFN- $\beta$ for another $24 \mathrm{~h}$. (B) LN-229 cells were pretreated or not with IFN- $\beta(100$ or $1,000 \mathrm{IU} / \mathrm{ml})$ for $24 \mathrm{~h}$ and then exposed to TG02 in the absence or presence of zVAD-fmk $(100 \mu \mathrm{M})$ for another $24 \mathrm{~h}$ prior to fluorimetric determination of DEVD-amc cleavage. (C) A similar experiment was conducted to compare the effects of IFN- $\beta$ and TG02 and their combination in $b c l-2$-transfected versus control transfected cells. In (B) and (C), stauro treatment for $24 \mathrm{~h}$ was used as a positive control. (D) LN-229 cells were treated as in Fig. $1 \mathrm{~A}$, but co-exposed to zVAD-fmk $(100 \mu \mathrm{M})$ during TG02 exposure. (E) Bcl-2-transfected vs. control transfected LN-229 cells were pretreated or not with IFN- $\beta$ (100 or $1,000 \mathrm{IU} / \mathrm{ml}$ ) for $24 \mathrm{~h}$ and then exposed to TG02. Metabolic activity was assessed at $72 \mathrm{~h}$ by MTT assay. Casp 3, caspase 3; IFN, interferon; stauro, staurosporine; ac-DEVD-amc, acetyl-Asp-Glu-Val-Asp-7-amino-4-methyl coumarin; zVAD-fmk, benzyloxycarbonyl-Val-Ala-Asp-fluoromethylketone; a.u., arbitrary units.

nor BCL-2 interfered specifically with the sensitizing properties of IFN- $\beta$ for TG02-induced cell death (Fig. 3D and E). This challenges the view that the enhanced caspase activity demonstrated in Fig. 3A-C was responsible for synergistic growth inhibition.
Neither xaf nor caspase 3 gene silencing block IFN- $\beta$ induced sensitization to TG02. To determine in more depth whether caspase-dependent apoptotic signalling mediates the synergy of IFN- $\beta$ and TG02, we explored whether $x a f$ induction or expression of caspase 3 were required for IFN- $\beta$-mediated 
A
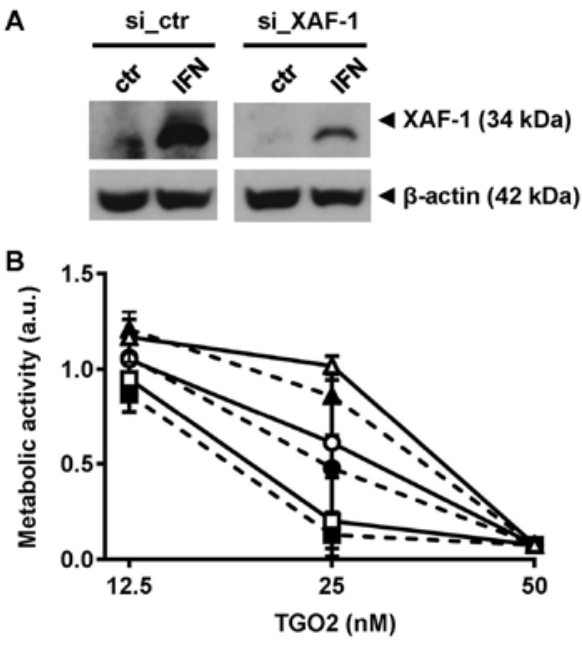

$\triangle \mathrm{scr}, \mathrm{TG02}$

- $-\mathrm{scr}, \mathrm{TG} 02+10 \mathrm{IU} / \mathrm{ml} \mathrm{IFN}$

ㅁ. scr, TG02 + $100 \mathrm{IU} / \mathrm{ml}$ IFN

$\pm \mathrm{XAF}-1, \mathrm{TG} 02$

- XAF-1, TG02 + $10 \mathrm{IU} / \mathrm{ml} \mathrm{IFN}$

- XAF-1, TG02 + $100 \mathrm{IU} / \mathrm{ml}$ IFN

C
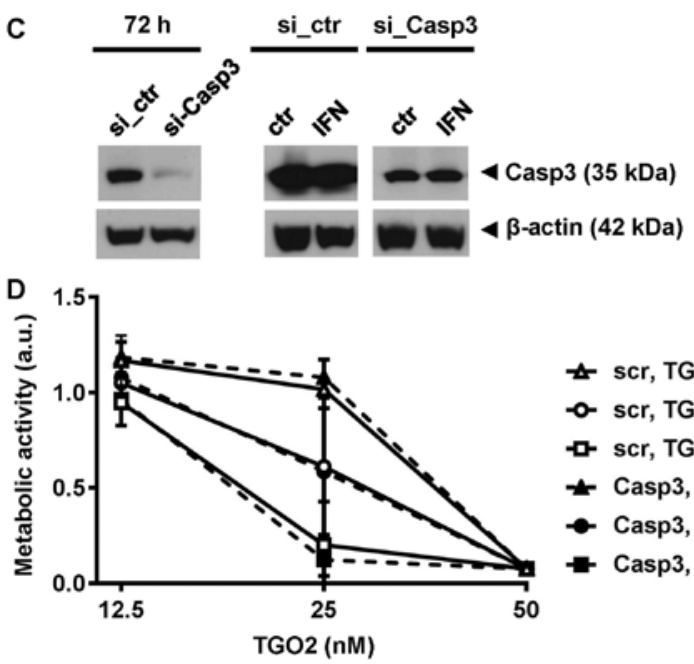

$\triangle \mathrm{scr}, \mathrm{TG} 02$

- - scr, $\mathrm{TG} 02+10 \mathrm{IU} / \mathrm{ml}$ IFN

ㅁ. scr, TG02 + $100 \mathrm{IU} / \mathrm{ml}$ IFN

¿ Casp3, TG02

- Casp3, TG02 + 10 IU/ml IFN

- Casp3, TG02 + $100 \mathrm{IU} / \mathrm{ml}$ IFN

Figure 4. IFN- $\beta$-mediated sensitization does not require XAF-1 or caspase 3. (A) XAF-1 protein levels were determined with or without IFN- $\beta 1$ a stimulation $(1,000 \mathrm{IU} / \mathrm{ml})$ for $24 \mathrm{~h}$ started at $72 \mathrm{~h}$ after electroporation to induce XAF-1 silencing in ZH-161 cells. (B) ZH-161 were pretreated or not with IFN- $\beta$ at 10 or $100 \mathrm{IU} / \mathrm{ml}$ for $24 \mathrm{~h}$ and then exposed to increasing concentrations of TG02. Metabolic activity was assessed after $>10$ days by MTT assay. (C) Caspase 3 gene silencing in ZH-161 cells assessed by immunoblot at $72 \mathrm{~h}$, maintained in the presence of IFN- $\beta$ [(1,000 IU/ml) for $24 \mathrm{~h}]$. $\beta$-actin served as loading control. (D) Control-transfected or caspase 3-silenced ZH-161 cells treated as in (B). XAF, XIAP-associated factor; si, small interfering RNA; ctr, control; IFN, interferon; Casp3, caspase 3; a.u., arbitrary units; scr, scrambled control.

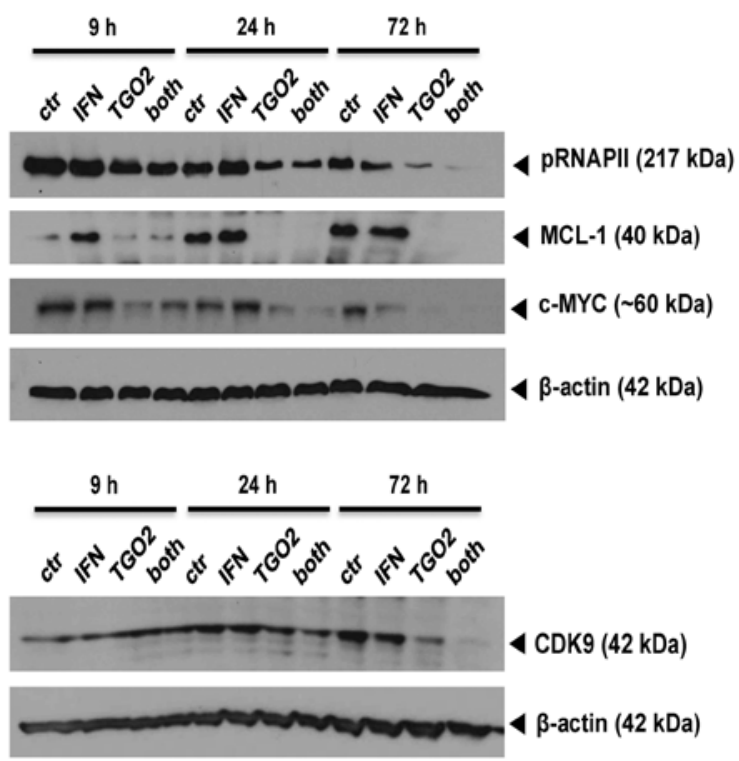

Figure 5. IFN- $\beta$ facilitates TG02-induced inhibition of RNA polymerase II phosphorylation. Levels of pRNAPII, MCL-1, c-MYC and CDK9 were determined in LN-229 cells pretreated or not with IFN- $\beta(1,000 \mathrm{IU} / \mathrm{ml})$ for $24 \mathrm{~h}$ and then exposed to TG02 (100 nM) for 9, 24 or $72 \mathrm{~h}$. $\beta$-actin served as loading control. pRNAPII, phosphorylated RNA polymerase II; ctr, control; IFN, interferon. 
sensitization to TG02. XAF is a previously identified IFN- $\beta$ response gene in human glioma cells including GIC (10) (Fig. S2A). We silenced xaf expression (Fig. S2B) which led to a strong reduction of XAF protein in response to IFN- $\beta$ (Fig. 4A). However, depletion of XAF did not abrogate the sensitization of glioma cells to TG02 mediated by pre-exposure to IFN- $\beta$ (Fig. 4B). Similarly, depletion of caspase 3 failed to affect the activity of this combination (Fig. 4C and D). We had previously observed features of senescence in one of two long-term glioma cell lines chronically exposed to TG02 (12). Accordingly, we determined senescence-associated $\beta$-galactosidase activity in two GIC models upon exposure to IFN- $\beta$ or TG02 or both, using irradiation at $20 \mathrm{~Gy}$ as a positive control (19). S-24 cells stimulated with IFN- $\beta$ or TG02 showed no increase in $\beta$-galactosidase activity whereas co-treatment did, but no such effect was seen in ZH-161 cells (Fig. S3).

IFN- $\beta$ facilitates direct TG02 target inhibition. Altogether these data indicated that the synergistic growth inhibition mediated by pre-exposure to IFN- $\beta$ followed by TG02 treatment might operate further up-stream, prior to the cell death pathways involving caspase inhibition or senescence. Indeed, immunoblot analyses allowed to demonstrate a time-dependent suppression of RNA polymerase II phosphorylation by TG02 that was facilitated in glioma cells pre-exposed to IFN- $\beta$. Yet, the TG02-induced decreases of two major putative down-stream effectors of TG02, MCL-1 and c-MYC, were not enforced by IFN- $\beta$. In contrast, the protein levels of CDK9, the bona fide main target of TG02 and the kinase responsible for RNA polymerase II phosphorylation, were decreased by TG02 alone and more so by the combination, at $72 \mathrm{~h}$ (Fig. 5).

\section{Discussion}

Effective pharmacological treatment options for glioblastoma have not been developed since the introduction of temozolomide in $2005(5,6)$. Immunotherapy may have failed because glioblastomas are currently considered as lymphocyte-depleted or cold tumors (20). Targeted therapy has not been successful because glioblastoma in most instances, if not all, is not a single pathway-driven disease (7). This provides space for the development of innovative, more broadly acting anti-cancer agents in glioblastoma. A prominent example currently explored in a pivotal trial is the proteasome inhibitor, marizomib (NCT03345095). Another example is TG02, an orally available inhibitor of multiple CDK with good blood brain barrier penetration (NCT03224104). This drug has shown promising single agent activity at least in cell culture models $(12,13)$.

We report that pre-exposure to type I interferon sensitizes human glioma models including GIC cultures to subsequent exposure to TG02, both in acute growth inhibition and in clonogenic survival assays (Fig. 1). We hypothesized that this synergy was related to the promotion of caspase-dependent apoptosis since IFN- $\beta$ induces a shift in gene expression that should facilitate caspase-mediated cell death (10). Furthermore, TG02 alone is not a potent activator of the caspase-dependent cell death pathway, but activates alternative death pathways (12). Consistent with the expectation, immunoblot and caspase activity assays confirmed synergy in promoting caspase activation when IFN- $\beta$ and TG02 were combined. Consistent with a mitochondrial type of caspase-mediated cell death, overexpression of BCL2 was protective (Fig. 3). A likely candidate promoting synergistic cell death was the proapoptotic protein, XAF1, which is highly inducible by type I interferon (10). Yet, a role of XAF1 in mediating synergistic cell death induction was excluded by XAF gene silencing. Further, somewhat unexpectedly, gene silencing of caspase 3 did not abrogate the synergy of type I interferon and TG02 either, and, expectedly, also not the activity of either agent administered alone (Fig. 4).

Finally, we reconsidered that potentially synergy might operate more up-stream at the level where TG02 proximately triggers the cell death cascade. Indeed, immunoblot confirmed that there was a time-dependent decrease in the phosphorylation of RNAPII which was greatly facilitated by pre-exposure to IFN- $\beta$ (Fig. 5). This loss of pRNAPII is predicted in response to TG02 since RNAPII is phosphorylated by CDK9, the principal target of TG02. That total CDK9 protein was also decreased, points to $c d k 9$ being one of the genes with short-lived expression that is subject to TG02-induced transcriptional perturbations. Exploring how IFN signaling primes glioma cells for TG02-mediated direct target inhibition may help to design novel, more effective pharmacological approaches to glioblastoma.

\section{Acknowledgements}

Not applicable.

\section{Funding}

The present study was supported by an unrestricted grant from Adastra to MW and by a Swiss National Science Foundation grant to MW (grant no. 31003A_166634/1).

\section{Availability of data and materials}

The datasets used and/or analyzed during the current study are available from the corresponding author on reasonable request.

\section{Authors' contributions}

ELR and MW conceived and supervised the study. BL, ELR and MW designed experiments. BL, ELR, MS and ME performed experiments. BL, ELR, MS and MW analysed data. MW wrote the first draft of the manuscript. BL and ELR made manuscript revisions. All authors approved the final version of the manuscript.

\section{Ethics approval and consent to participate}

The generation of human GIC was approved by the Ethics Committee of the Canton of Zurich, Switzerland (approval no. KEK 2016-00456). Informed consent was obtained from the patients from whose tumors the GIC lines were generated.

\section{Patient consent for publication}

Not applicable. 


\section{Competing interests}

MW received an unrestricted research grant from Adastra (San Diego, CA). The other authors declare that they have no competing interests.

\section{References}

1. Ostrom QT, Gittleman H, Truitt G, Boscia A, Kruchko C and Barnholtz-Sloan JS: CBTRUS statistical report: Primary brain and other central nervous system tumors diagnosed in the united states in 2011-2015. Neuro Oncol 20 (Suppl-4): iv1-iv86, 2018.

2. Weller M, Wick W, Aldape K, Brada M, Berger M, Pfister SM Nishikawa R, Rosenthal M, Wen PY, Stupp R and Reifenberger G: Glioma. Nat Rev Dis Primers 1: 15017, 2015.

3. Louis DN, Perry A, Reifenberger G, von Deimling A Figarella-Branger D, Cavenee WK, Ohgaki H, Wiestler OD, Kleihues P and Ellison DW: The 2016 World Health Organization Classification of Tumors of the Central Nervous System: A summary. Acta Neuropathol 131: 803-820, 2016.

4. Brennan CW, Verhaak RGW, McKenna A, Campos B, Noushmehr H, Salama SR, Zheng S, Chakravarty D, Sanborn JZ, Berman SH, et al: The somatic genomic landscape of glioblastoma. Cell 155: 462-477, 2013

5. Stupp R, Mason WP, van den Bent MJ, Weller M, Fisher B Taphoorn MJ, Belanger $\mathrm{K}$, Brandes AA, Marosi C, Bogdahn U, et al: Radiotherapy plus concomitant and adjuvant temozolomide for glioblastoma. N Engl J Med 352: 987-996, 2005.

6. Weller M, van den Bent M, Tonn JC, Stupp R, Preusser M, Cohen-Jonathan-Moyal E, Henriksson R, Le Rhun E, Balana C, Chinot O, et al: European association for neuro-oncology (EANO) guideline on the diagnosis and treatment of adult astrocytic and oligodendroglial gliomas. Lancet Oncol 18: e315-e329, 2017.

7. Prados MD, Byron SA, Tran NL, Phillips JJ, Molinaro AM, Ligon KL, Wen PY, Kuhn JG, Mellinghoff IK, de Groot JF, et al: Toward precision medicine in glioblastoma: The promise and the challenges. Neuro Oncol 17: 1051-1063, 2015.

8. Yue C, Xu J, Tan Estioko MD, Kotredes KP, Lopez-Otalora Y, Hilliard BA, Baker DP, Gallucci S and Gamero AM: Host STAT2/type I interferon axis controls tumor growth. Int J Cancer 136: 117-126, 2015.

9. Du Z, Cai C, Sims M, Boop FA, Davidoff AM and Pfeffer LM: The effects of type I interferon on glioblastoma cancer stem cells. Biochem Biophys Res Commun 491: 343-348, 2017.
10. Happold C, Roth P, Silginer M, Florea AM, Lamszus K, Frei K, Deenen R, Reifenberger $G$ and Weller M: Interferon- $\beta$ induces loss of spherogenicity and overcomes therapy resistance of glioblastoma stem cells. Mol Cancer Ther 13: 948-961, 2014.

11. Wakabayashi T, Natsume A, Mizusawa J, Katayama H, Fukuda H, Sumi M, Nishikawa R, Narita Y, Muragaki Y, Maruyama T, et al: JCOG0911 INTEGRA study: A randomized screening phase II trial of interferon $\beta$ plus temozolomide in comparison with temozolomide alone for newly diagnosed glioblastoma. J Neurooncol 138: 627-636, 2018.

12. Le Rhun E, von Achenbach C, Lohmann B, Silginer M, Schneider H, Meetze K, Szabo E and Weller M: Profound, durable and MGMT-independent sensitivity of glioblastoma cells to cyclin-dependent kinase inhibition. Int J Cancer 145: 242-253, 2019.

13. Su YT, Chen R, Wang H, Song H, Zhang Q, Chen LY, Lappin H, Vasconcelos G, Lita A, Maric D, et al: Novel targeting of transcription and metabolism in glioblastoma. Clin Cancer Res 24: 1124-1137, 2018.

14. Wu J, Bryla C, McCoy A, Lisa B, Garren N, Siegel C, Grajkowska E, Theeler B, Park DM, Parrott T, et al: ACTR-69. Phase I trial of TG02 plus dose-dense or metronomic temozolomide for adults with recurrent anaplastic astrocytoma and glioblastoma. Neuro Oncol 19 (suppl-6): vi15-vi15, 2017.

15. Livak KJ and Schmittgen TD: Analysis of relative gene expression data using real-time quantitative PCR and the 2(-Delta Delta C(T)) method. Methods 25: 402-408, 2001.

16. Greco WR, Bravo G and Parsons JC: The search for synergy: A critical review from a response surface perspective. Pharmacol Rev 47: 331-385, 1995.

17. Silginer M, Nagy S, Happold C, Schneider H, Weller M and Roth P: Autocrine activation of the IFN signaling pathway may promote immune escape in glioblastoma. Neuro Oncol 19: 1338-1349, 2017.

18. Weller M, Trepel M, Grimmel C, Schabet M, Bremen D, Krajewski S and Reed JC: Hypericin-induced apoptosis of human malignant glioma cells is light-dependent, independent of bcl-2 expression, and does not require wild-type p53. Neurol Res 19: 459-470, 1997.

19. Fumagalli M, Rossiello F, Mondello $C$ and d'Adda di Fagagna F: Stable cellular senescence is associated with persistent DDR activation. PLoS One 9: e110969, 2014.

20. Lim M, Xia Y, Bettegowda C and Weller M: Current state of immunotherapy for glioblastoma. Nat Rev Clin Oncol 15: 422-442, 2018.

This work is licensed under a Creative Commons Attribution-NonCommercial-NoDerivatives 4.0 International (CC BY-NC-ND 4.0) License. 